\title{
THE IMPACT OF PROBIOTIC ADDITIVE ON SOME CELL BLOOD COMPOSITION AND ON CHOLESTEROL IN LAMBS
}

\author{
MOUSA AL HAMOUD; SHARIF SHAHIN and AHMED H. MOKRESH \\ Faculty of Veterinary Medicine, Albaath University. \\ Email: rosealhamod@yahoo.com
}

\section{ABSTRACT}

Received at: 31/12/2013

This study was conducted to evaluate the effect of Probiotic (primalac ${ }^{\circledR}$ ) on Cell Blood picture and on Cholesterol in lambs. Thirty lambs from the Syrian Awassi lambs, aged approximately 5 months with average body weight $(32 \pm 0.7 \mathrm{~kg})$ Were used in this experiment. The experiment of animals were devided into three groups: Accepted: 3/3/2014 (T0, T1 and T2). All groups were fed on a traditional diet each animal of group T1 was received, in addition to this traditional diet, $2 \mathrm{~g}$ of Probiotic, and animal of group $\mathrm{T} 2$ were received $4 \mathrm{~g}$ of Probiotics for each. Blood was collectedfrom the groups at the endofthe experiment tocarry blood picture (RBC, WBC, PCV, HB, DWBC and Cholesterol). The results of the search to a bloody and Cholesterol changes in the groups compared to the control. Our results showed the following: An increase in the value of (RBC, HB, PCV) compared to the control, but did not reach the increase to the level of significance. (WBC, DWBC) was not affected by adding probiotics to the diet of the Lambs. A significant decrease in the concentration of The cholesterol in groups $(\mathrm{T} 1, \mathrm{~T} 2)$ compared to the control $(\mathrm{P}<0.05)$.

Key Words: Primalac ${ }^{\circledR}$, Probiotic, Awassi lambs, Cholesterol, Cell Blood Composition, total differental leukocytescount.

$$
\begin{aligned}
& \text { تأثير إضافة البروبيوتيك على بعض مكونات الام الخلوية والكولسترول عند الحملان } \\
& \text { موسى الحمود ، شريف شاهين ، أحمد حمدي مقرش }
\end{aligned}
$$

أجري البحث لدر اسة تأثير إضافة البروبيوتيك (البريمالاك) على بعض مكونات الدم الخلوية و الكولسترول عند الحملان ، حيث استخدم في هذه

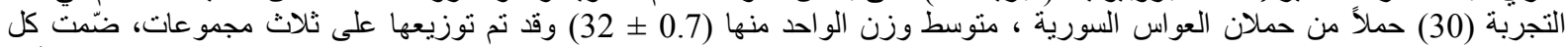

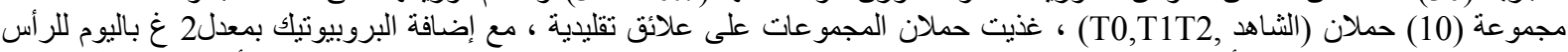
للمجموعة T1 و4 (10) باليوم اللرأس للمجموعة T2 ولمدة شهرين منتالين. نم سحب الدم من المجموعات في نهاية التجربة لأجر اء التحاليل الدموية

.(RBC, WBC, DWBC, PCV, HB, Cholesterol)

أثنارت نتائج البحث إلى حدوث تغير ات دموية وبيوكيميائية في المجموعات مقارنة بالثاهد الأمر الذي يعزز ويؤكد دور البروبيوتيك في تربية

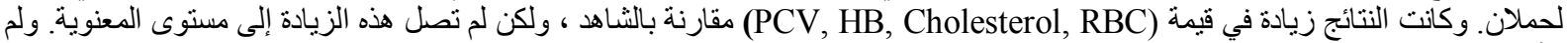

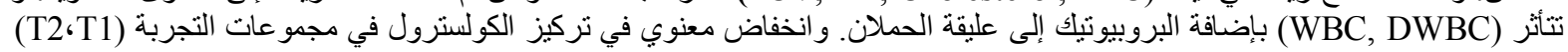

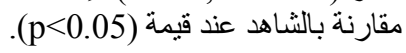

\section{INTRODUCTION المقدمـة}

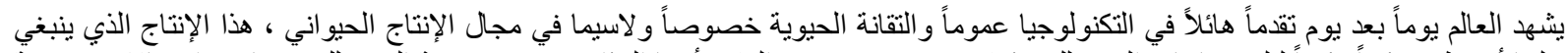

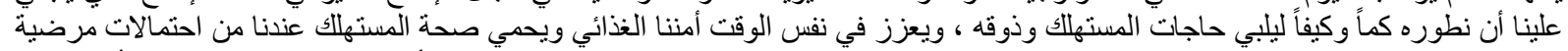

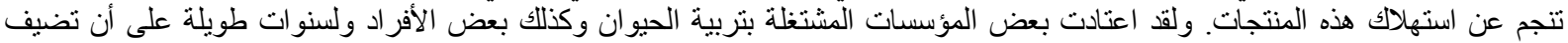

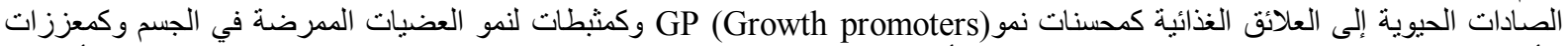

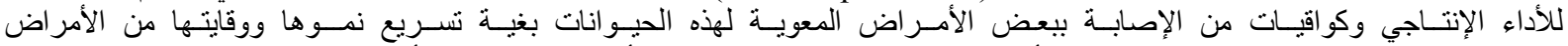
(Truscott and Alsheikhly, 1977) للاستهلاك البشري و السبب في ذللك عائد إلى ثمالاتها (Residues) المضرة وظهور أنواع من البكثيريا المقاومة للعديد من الصادات في الحيوية في آن
} 
واحد (Philips,1999). ولذلك قامت منظمة الصحة العالمية باستصدار تعليمات ونصائح تمنع إضافة بعض الصادات الحيوية إلى العليقة الغذائية

.(Woodward, 2005)

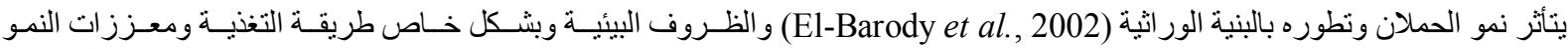
حيث تؤثر التغذية بشكل مفيد في إنتاج حملان تجارية من خلال إذخال (Abd El-Gawad et al., 2002, Andrighetto et al., 1993)

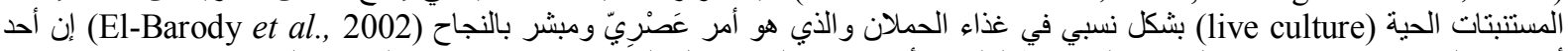

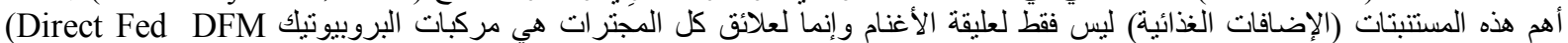

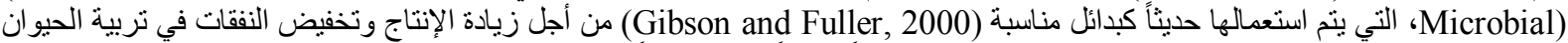

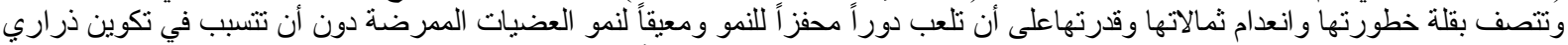

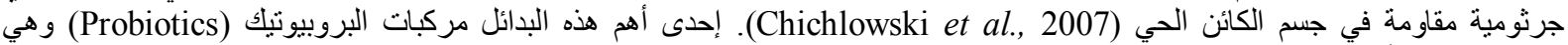

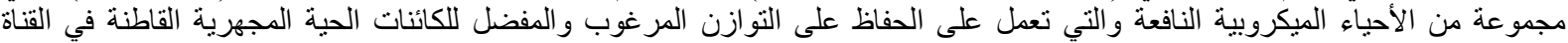

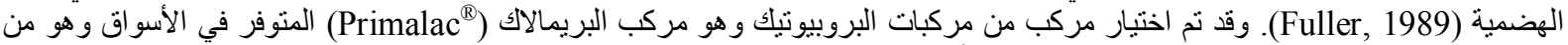

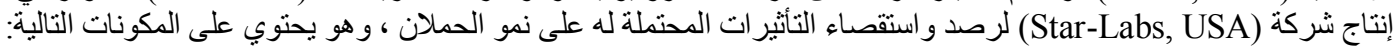

- 1 x $10^{9} \mathrm{CFU} / \mathrm{Gram}=$ Billion Organisms/g

- Lactobacillus acidophilus fermentation product dehydrated $2.5 \times 10^{8}=250,000,000$

- Lactobacillus casei fermentation product dehydrated $2.5 \times 10^{8}=250,000,000$

- Bifidobacteriumbifidium fermentation product dehydrated $2.5 \times 10^{8}=250,000,000$

- Enterococcus faecium fermentation product dehydrated 2.5 x $108=250,000,000$

- Rice mill by product

- Calcium carbonate

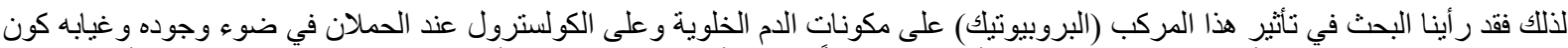

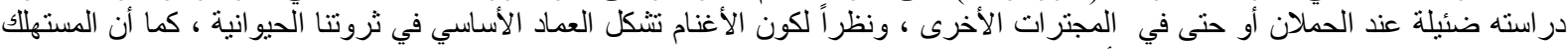
عندنا يفضل لحمها على لحوم الحيو انات الأخرى الموجودة في القطر.

\section{OBJECTIVES}

أهداف البحث

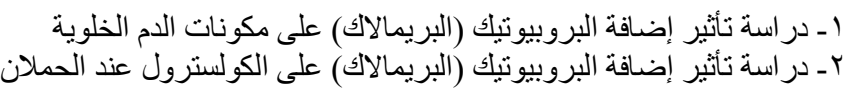

\section{MATERIALS and METHODS \\ مواد وطر ائق البحث}

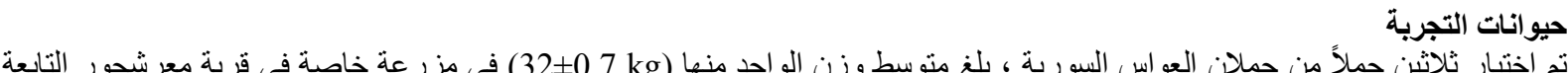

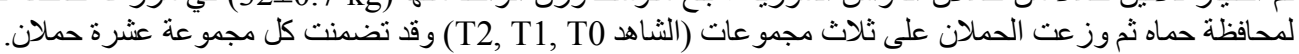

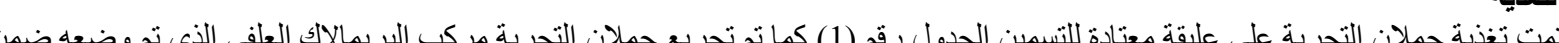

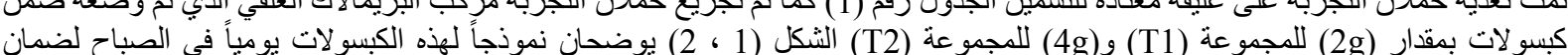
وصول المركب إلى كل حيو ان من حيو انات المجموعة الو احدة وبالجر عة المطلوبة حسب خطة التجربة الموضحة في الجدول رقم(2).

$$
\begin{aligned}
& \text { شكل رقم ץ : نموذج للكبسو لات } \\
& \text { المعطاة للمجمو عة T2 المحتوية 4g بريمالاك لاك }
\end{aligned}
$$

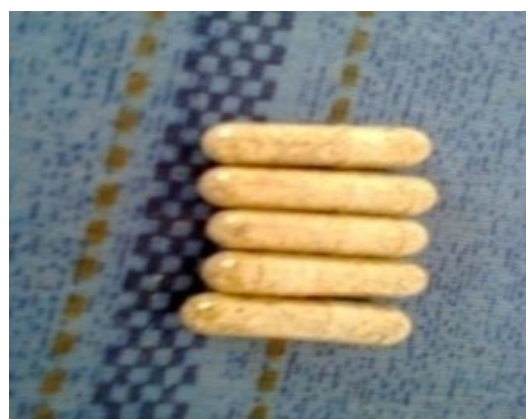

شكل رقم ا: نموذج للكبسو لات

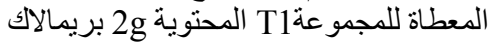

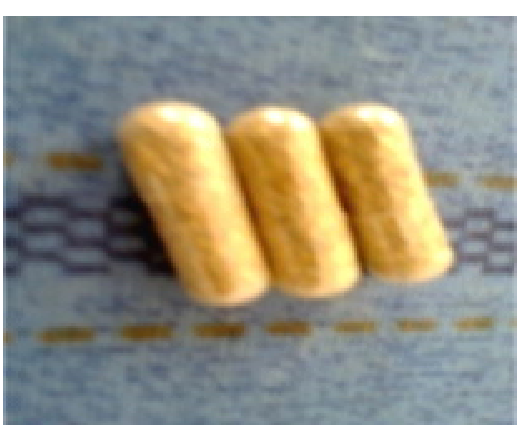


جدول 1: مو اد العلف الأولية المستعملة في تركيب العليقة الأساسية للتجربة \%\%.

\begin{tabular}{|c|c|}
\hline نسبة المادة العلفية المستعملة في العليقة \% & المادة العلقية \\
\hline 62 & شعير \\
\hline 17 & ذرة صفراء \\
\hline 2 & كسبة قطن مقشورة 44\% \\
\hline 3 & كسبة صويا 48\% \\
\hline 15 & 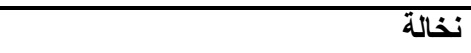 \\
\hline 0.4 & فوسفات ثنائية الكالسيوم \\
\hline 0.5 & ملح طعام \\
\hline 0.1 & فيتامينات وأملاح \\
\hline 100 & المجموع \\
\hline
\end{tabular}

جدول r : مجموعات التجربة ووزن جر عة البريمالاك المضافة يو مياً.

\begin{tabular}{|c|c|c|}
\hline جرعة البريمالاك & عدد الحملان & مجموعات التجربة \\
\hline- & 10 & T0 \\
\hline 2g للرأس يوميا & 10 & T1 \\
\hline 4g للرأس يوميا & 10 & T2 \\
\hline
\end{tabular}

جمع عينات الام والتحاليل المجراة عليها

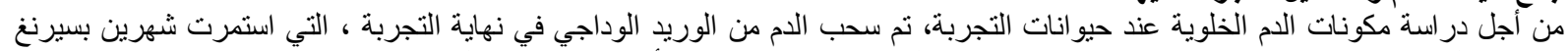

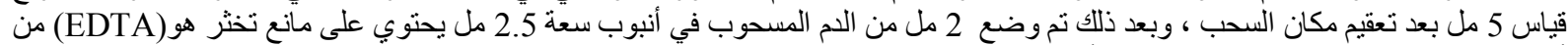

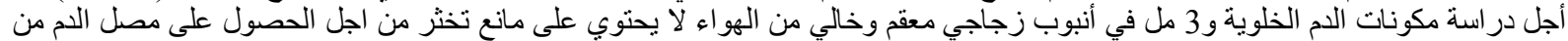
أجل در اسة الكولسترول (Cholesterol)

• تم تقدير قيمة (PCV \% بطريقة الأنابيب الثعرية Microhematocrit - method باستخدام أنابيب دقيقة طولها 75ملم وقطر هـ/1/ ملم ومفتوحة الطرفين وخالية من أي مادة مانعة للتخثر.

• تم تقدير التعداد الكلي للكريات الدموية الحمر اء RBC و البيضاء WBC بطريقة العد التقليدية وباستخدام عدادة نيوباور المعدلة.

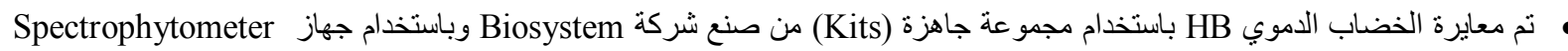

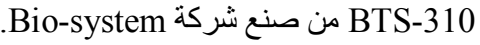

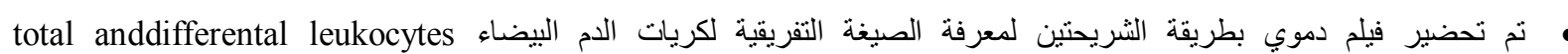
(countDWBC)

• تم معايرة الكولسترول Cholesterol باستخدام مجموعة كيت جاهزة وحسب توصيات الثركة المصنعة (Human) وباستخدام جهاز Spectophotometer

التحليل الإحصائي: Statistical analysis للاحليل الإحصائي للمعطيات تم استخدام برنامج حاسوبي من نوع (SPS14.0) حيث قمنا بتحليل التباين لمعيار واحد (One Way ANOVA).

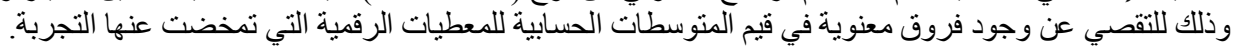




\section{RESULTS \\ النتائج}

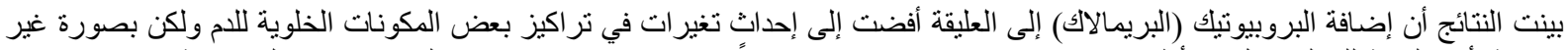

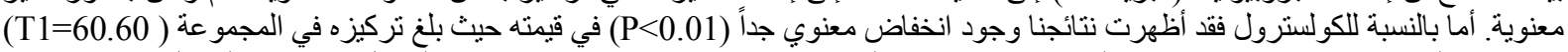

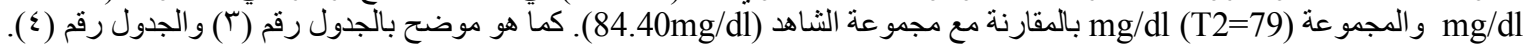

$$
\text { جدول رقم r: تأثير البريمالاك على مكونات الدم الخلوية والكولسترول عند الحملان. }
$$

\begin{tabular}{|c|c|c|c|}
\hline \multicolumn{3}{|c|}{ مجموعات التجربة } & \multirow{3}{*}{ المؤشرات } \\
\hline T2 & $\mathrm{T} 1$ & T0 الثاهد T0 & \\
\hline Mean \pm SD & Mean \pm SD & Mean \pm SD & \\
\hline $8.74 \pm 0.86$ & $9.19 \pm 0.64$ & $8.79 \pm 0.71$ & $\begin{array}{c}\mathrm{RBC}^{2} \times 10^{6} \\
\mu \mathrm{L}\end{array}$ \\
\hline $9.16 \pm 0.40$ & $9.04 \pm 0.39$ & $9.42 \pm 0.48$ & $\mathrm{WBC} \times 10^{3} \mu \mathrm{L}$ \\
\hline $9.74 \pm 0.61$ & $9.96 \pm 0.26$ & $9.60 \pm 0.56$ & HB $\mathrm{g} / \mathrm{dl}$ \\
\hline $29.20 \pm 2.16$ & $29.40 \pm 1.14$ & $29.8 \pm 1.78$ & $\begin{array}{c}\text { PCV } \\
\%\end{array}$ \\
\hline $\begin{array}{c}79 \pm 5.78 \\
\text { b }\end{array}$ & $\begin{array}{c}60.60 \pm 19.32 \\
\mathrm{Aa}\end{array}$ & $\begin{array}{c}84.40 \pm 3.20 \\
\text { B }\end{array}$ & $\begin{array}{c}\text { Cholesterol } \\
\text { mg/dl }\end{array}$ \\
\hline
\end{tabular}

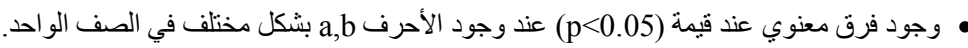

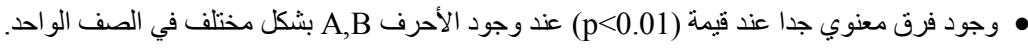

جدول رقم ؟ : تأثثر إضافة البريمالاك على الصيغة التفريقية للكريات البيض عند الحملان.

\begin{tabular}{|c|c|c|c|}
\hline \multicolumn{3}{|c|}{ مجموعات التجربة } & \multirow{3}{*}{ المؤشرات } \\
\hline $\mathbf{T 2}$ & T1 & T0 الثاهد & \\
\hline Mean \pm SD & Mean \pm SD & Mean \pm SD & \\
\hline $80.40 \pm 2.40$ & $77.80 \pm 3.34$ & $80.60 \pm 3.91$ & Lymphocyte \% \\
\hline $2.40 \pm 0.89$ & $2.60 \pm 0.89$ & $2.20 \pm 0.44$ & $\begin{array}{c}\text { Monocytes } \\
\%\end{array}$ \\
\hline $15.80 \pm 2.16$ & $18 \pm 2.91$ & $15.60 \pm 3.78$ & Neterophils \\
\hline $0.4 \pm 0.54$ & $0.4 \pm 0.54$ & $0.4 \pm 0.54$ & $\begin{array}{c}\text { Basophils } \\
\%\end{array}$ \\
\hline $1.2 \pm 0.44$ & $1.2 \pm 0.44$ & $1.2 \pm 0.44$ & $\begin{array}{c}\text { Eosinophils } \\
\%\end{array}$ \\
\hline
\end{tabular}

\section{DISCUSSION \\ المناقشة}

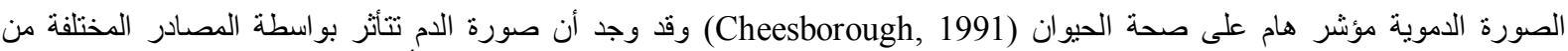

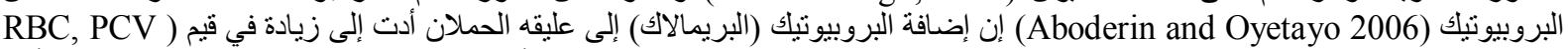

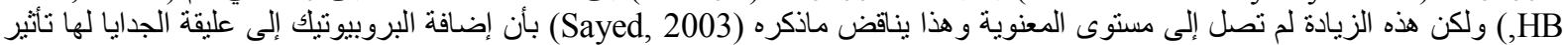

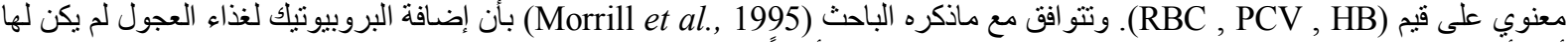
أي تأثير معنوي على تعداد كريات الدم الحمر اء واليضاء وتثو افتق أيضاً مع (Sadiek and Boehm, 2001) اللذان وجدا النتيجة نفسها عند

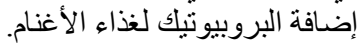

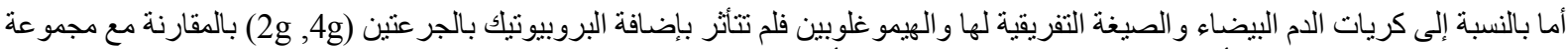

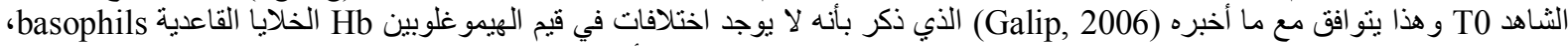

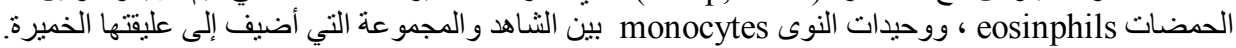


ويتو افق مع كلاً من (Aboderin and Oyetayo, 2006) حيث وجدا بأن وحيدات النوى monocytes والخلايا القاعدية basophils ونائ

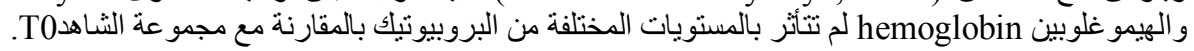

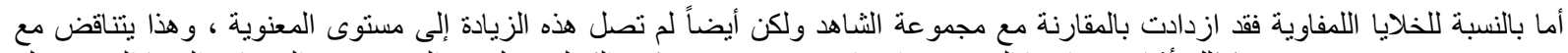
(Belewu et al., 2008)

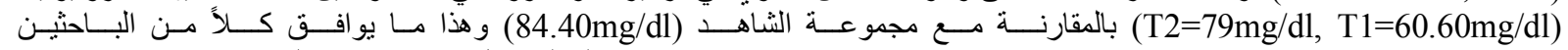
(Alp, and Kahraman, 1996, Lubbadeh et al., 1999)

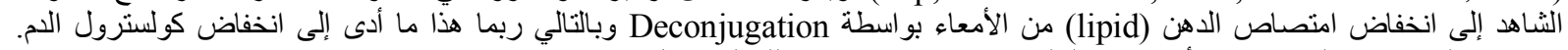
وربما تفكيك عصارة المرارة في الأمعاء ، وبالتالي منع إعادة الامنصاء بواصنة للكوليسترول.

ولكن يجب الاعتر اف بأن العثور على الأليات الفعالة التي تؤدي إلى خفض كوليسترول الدم تحت تأثثر إضافة البريمالاك إلى العليقة ، يحتاج إلى أبحاث رصينة أخرى نركز على هذا المنور على الاليات

\section{CONCLUSIONS and RECOMMENDATIONS} الاستنتاجات والتوصيات

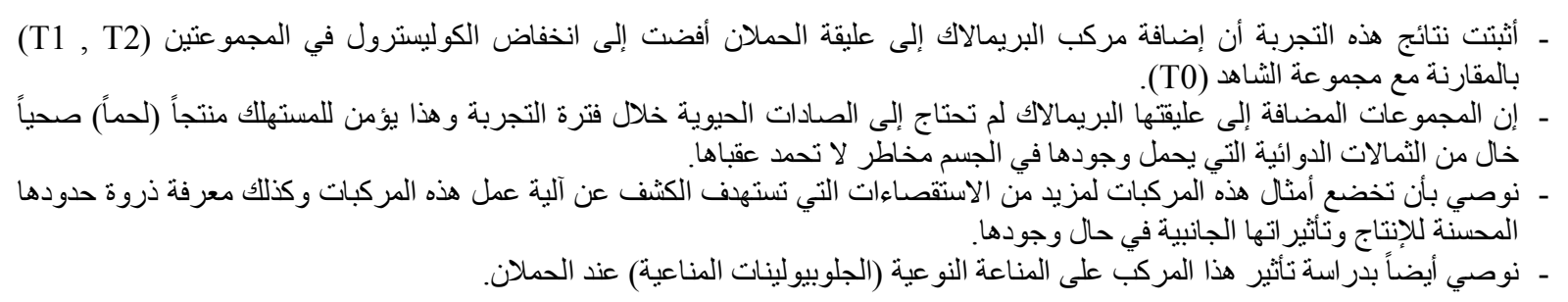

\section{REFERENCES}

المراجـ

Abd El-Gawad, Eman, I.; Maharm, G.M.; Faten, F. Abou Ammo and Fathia A. Ibrahim. (2002): Effect of yeast culture (Lacto-Sacc) supplementation on growth, some blood parameters and carcass quality of goats. Egypt. J. Appl. Sci., 17 (7): 375-388.

Aboderin, F.I. and Oyetayo, V.O. (2006): Haematological Studies of Rats Fed Different doses of Probiotics, Lactobacillus plantarum, Isolated from Fermenting Co Slurry. Pak. J. Nutr. 5(2): 102-105.

Alp, M. and Kahraman, R. (1996): Utilization of probiotics in animal nutrition. Istanbul Üniv. Vet. Fak. Derg., 22: $1-8$.

Andrighetto, J.; Bailoni, L.; Cozzi, G. and Berzaghi, P. (1993): Effect of yeast culture addition on digestion in sheep fed a highconcentrated diet. Small ruminant research.12.27-34.

Belewu, M.A.; Yahaya, A.A. and Adeyina, A.O. (2008): Study on some haematological parameters of goats fed aspergillus treated and untreated shea-butter cake. Rev. J. Anim. Sci. 2(5): 154-156.

Cheesborough, M. (1991): Medical laboratory manual for tropical countries. 2nd edition Tropical Health Technology and Butterworth Scientific limited. 1: 494-526.

Chichlowski, M., Croom, J.; McBride, B.W.; Havenstien, G.B. and Koci, M.D. (2007): Metabolic and physiological impact of Probiotics or direct -fed microbes on poultry: A brief review of current knowledge. Int. J. Poult. Sci. 6(10): 694-704.

El-Barody, M.A.A.; Abdalla, E.B. and Abd El-Hakim, A.A. (2002): The changes in some blood metabolites associated with the physiological responses in sheep. Livestock Prod. Sci., 75: 45-50.

El-Shamaa, I.S. (2002): Onset of puberty, semen production and blood constituents in crossbred male lambs as affected by dietaryyeast culture addition. J. Agric. Sci. Mansoura Univ., 27(7): 4589-4598.

Fuller, R. (1989): Probiotics in man and animals. J. Appl. acterial., 66: 365-378.

Galip, N. (2006): Effect of supplemental yeast culture and sodium bicarbonate on ruminal fermentation and blood variables in rams. J. Anim. Phys. and Anim. Nutri. 90: 446-452.

Gibson, G.R. and Fuller, R. (2000): Aspects of in vitro and in vivo research approaches directed toward identifying probiotics and prebiotics for human use. J. Nutr., 130: 391-395.

Lubbadeh, W.; Haddadin, M.S.Y.; Al-Tamimi, M.A. and Robinson, R.K. (1999): Effect on cholesterol contentof fresh lamb of supplementing the feed of Awassiewes and lambs with Lactobacillus acidophilus. Meat Sci., 52: 381-385. 
Morrill, J.L.; Morrill, J.M.; Feyerherm, A.M. and Laster, J.F. (1995): Plasma proteins and a probiotic as ingredients in milk replacer. J. Dairy Sci. 78: 902-907.

Philips, I. (1999): Assessing in the evidence that antibiotics growth promoters influence human infection. J. Hospital Infections, 43: 173-178

Sadiek, A. and Boehm, J. (2001): Influence of pronifer as a probiotic on the rumen fluid and blood parameters of sheep fed different roughage concentrate based diets. Wiener Tieraztliche Monatscshrift, 88: 4-10.

Sayed, A.S. (2003): Studies of the influence of pronifer as a probiotics on the clinical, hematological and biochemical status of the goat's kids. Assiut. Vet. Med, J. 99(98): 131-143.

Truscott, R.B. and Alsheikhly, F. (1977): The production and treatment of necrotic enteritis in broilers. AM. J. Vet. Res. 38: 857-861.

Woodward, P. (2005): Impact of a ban on animal by- products and antibiotic growth promoters - European experience. Page 46-57. In: Proceeding, Thirty second annual Carolina Poultry Nutrition Conference, North Carolina, October, 26-27. 